\title{
Development of Polyphosphate Parameters for Use with the AMBER Force Field
}

\author{
KRISTIN L. MEAGHER, LUKE T. REDMAN, HEATHER A. CARLSON \\ Department of Medicinal Chemistry, College of Pharmacy, University of Michigan, \\ 428 Church St., Ann Arbor, Michigan 48109-1065
}

Received 17 September 2002; Accepted 23 December 2002

\begin{abstract}
Accurate force fields are essential for reproducing the conformational and dynamic behavior of condensed-phase systems. The popular AMBER force field has parameters for monophosphates, but they do not extend well to polyphorylated molecules such as ADP and ATP. This work presents parameters for the partial charges, atom types, bond angles, and torsions in simple polyphosphorylated compounds. The parameters are based on molecular orbital calculations of methyldiphosphate and methyltriphosphate at the RHF/6-31+G* level. The new parameters were fit to the entire potential energy surface (not just minima) with an RMSD of $0.62 \mathrm{kcal} / \mathrm{mol}$. This is exceptional agreement and a significant improvement over the current parameters that produce a potential surface with an RMSD of $7.8 \mathrm{kcal} / \mathrm{mol}$ to that of the ab initio calculations. Testing has shown that the parameters are transferable and capable of reproducing the gas-phase conformations of inorganic diphosphate and triphosphate. Also, the parameters are an improvement over existing parameters in the condensed phase as shown by minimizations of ATP bound in several proteins. These parameters are intended for use with the existing AMBER 94/99 force field, and they will permit users to apply AMBER to a wider variety of important enzymatic systems.
\end{abstract}

(C) 2003 Wiley Periodicals, Inc. J Comput Chem 24: 1016-1025, 2003

Key words: molecular mechanics; calculations; computer simulation; GTP; nucleic acids

\section{Introduction}

The simulation of biological molecules is dependent on accurate and reliable force fields that reproduce the proper observed conformational behavior in the condensed phase. To enable simulations of large biomolecules, empirical potential energy functions have been created using molecular mechanics principles. These potential functions have been developed over the past four decades to allow for quick and accurate simulation of amino acid residues and nucleic acids. Although the inclusion of polarizable functions is a major emphasis in force field development, ${ }^{1}$ such attempts are in their infancy, and two-body potential functions are still widely used. Improvements and additions to traditional force fields are needed as simulations of more complicated biological systems reveal limitations in the current parameter set.

One of the most commonly used potential functions is the AMBER force field $;^{2}$ it is well parameterized for amino acids and nucleic acids. The force field includes parameters for monophosphates, allowing for the simulation of the nucleoside and nucleotide oligomers, but parameters for polyphosphates are missing. One study has presented highly specialized parameters for nucleotide sugars, ${ }^{3}$ but they do not extend well to alternate systems and cannot accurately reproduce the behavior of nucleoside polyphosphates like ATP. Parameters for polyphosphorylated mole- cules exist in force fields such as $\mathrm{CHARMM}^{4}$ and MM $3,{ }^{5}$ and those parameters are also needed for AMBER 94/99. The AMBER force field has estimated parameters for the torsions in polyphosphates, but to our knowledge, there has not been a systematic parameterization of these functional groups.

Polyphosphorylated molecules are ubiquitous in biochemistry. They include such diverse molecules as nucleotide triphosphates (ATP, GTP), nucleotide diphosphates (ADP, GDP), nicotinamide adenine dinucleotide (NAD+), and UDP-glucose. Specifically, the energy of ATP hydrolysis is harnessed to drive many enzymatic reactions, ${ }^{6}$ and the phosphorylation state of GTP is critical for the signaling cascades controlled by the G-protein coupled receptors. ${ }^{7}$

Correspondence to: H. A. Carlson; e-mail: carlsonh@umich.edu Contract/grant sponsors: Beckman Young Investigator Program Contract/grant sponsor: National Institutes of Health; contract/grant number: GM65372

Contract/grant sponsor: Pharmaceutical Sciences Training Program NIH Grant; contract/grant number: GM07767

This article includes Supplementary Material available from the authors upon request or via the Internet at ftp://ftp.wiley.com/public/journals/ $\mathrm{jcc} /$ suppmat/24/1016 or http://www.interscience.wiley.com/jpages/01928651/suppmat/v24.1016.html 


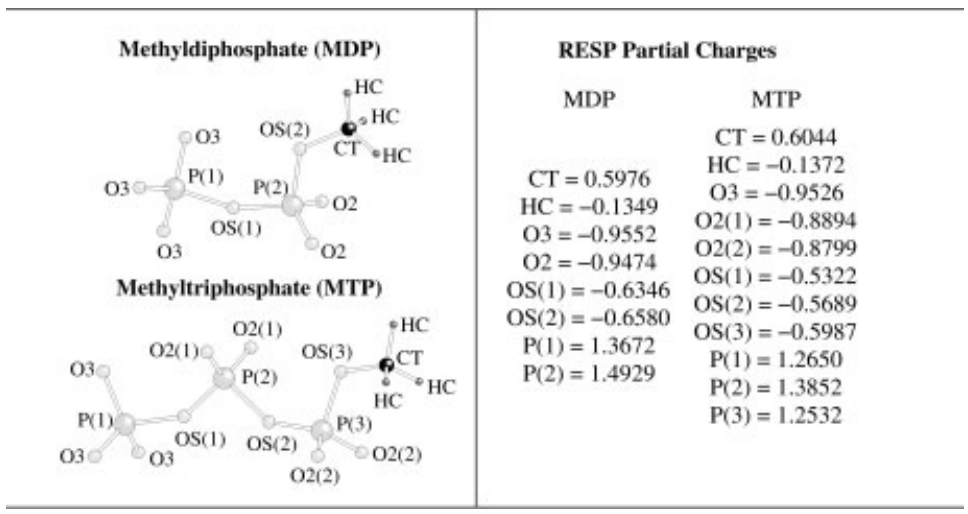

Figure 1. Extended conformations of MDP and MTP. Atom types and partial charges are also shown.

Accurate force field parameters for polyphosphorylated molecules are thus critical for the computational understanding of such systems.

Here, we report new parameters for use with the AMBER 94/99 potential function. These parameters extend the utility of the AMBER force field by providing more accurate simulations of phosphorylated biomolecules.

\section{Methods}

The modular nature of the AMBER force field allowed us to create polyphosphorylated nucleic acids through developing polyphosphate "tails" for the 5 ' position on the ribose ring. To model the conformational and energetic behavior of the polyphosphate groups, two simple model systems were chosen: methyl diphosphate (MDP) and methyl triphosphate (MTP). MDP and MTP have the computational advantage of being small, and they contain a methyl group that facilitates combining these new parameters with the existing AMBER parameters for the ribose ring.

Our procedure outlined below is based on the original development of the AMBER 94 force field, ${ }^{2}$ and every effort has been made to maintain the same protocol in creating these new parameters. It is possible that higher levels of theory and larger basis sets would be more appropriate for a highly charged system like this; however, the original calculations used by Cornell et al. ${ }^{2}$ were done at the RHF/6-31G* level. We chose to use RHF/6-31+G* to be consistent with previous parameterization and yet add the polarization functions necessary for these charged molecules.

\section{Generating the Potential Energy Surface for MDP and MTP}

Our initial $a b$ initio molecular orbital calculations were based on the conformations of MDP and MTP determined at the RHF/6$31 \mathrm{G}^{*}$ level by Hwang et al. ${ }^{8}$ In the gas phase, the lowest energy conformations of MTP and MDP contain an intramolecular "hydrogen bond" interaction between a terminal anionic oxygen and a hydrogen of the methyl group. This interaction is unlikely in biological systems due to the presence of solvent molecules and counter ions. To reduce the bias of these less-relevant regions in our search of the potential surface, we chose to focus on the more extended conformations for both MDP and MTP (Fig. 1). Previous quantum mechanical studies of these molecules did not include polarization functions, but the highly charged nature of MDP and MTP calls for the inclusion of such functions in the basis set. In this study, the conformational minima of MDP and MTP were calculated at the RHF/6-31+ $\mathrm{G}^{*}$ level using Gaussian 98. ${ }^{9}$

The minimum potential energy surface was then scanned in $10^{\circ}$ increments with respect to each torsional motion. The partial optimizations with respect to each torsion were calculated for the entire conformational space of the molecule (also at the RHF/ $6-31+\mathrm{G}^{*}$ level). Parameterization included the entire potential surface, not just the local minima. Additional local minima were identified from the torsional scans. These conformers were refined with additional energy minimizations. For some shallow minima ( 2 and $\mathbf{3}$ in Fig. 3), the two convergence criteria were relaxed. The maximum displacement was changed from the default of 0.00180 to $0.01 \AA$, and the RMS displacement was changed from 0.001200 to $0.006667 \AA$. Figures 2 and 3 give all minima determined for MDP and MTP, respectively.

\section{Parameterization Strategy}

The parameterization protocol outlined by Cornell et al. ${ }^{2}$ was followed to allow seamless incorporation of the new parameters. Partial charges were computed using the RESP methodology developed by Kollman et al. ${ }^{10}$ The total charge on the methyl group was constrained to $+0.19 e$ to allow incorporation into the existing force field. The functional form of the AMBER force field is given in eq. (1). The internal energy of a molecule is broken down into components for bond stretching, angle bending, torsional twisting, and nonbonded electrostatic and Lennard-Jones terms.

$$
\begin{aligned}
E=\sum_{\text {bonds }} K_{r}\left(r-r_{\mathrm{O}}\right)^{2} & +\sum_{\text {angles }} K_{\theta}\left(\theta-\theta_{\mathrm{O}}\right)^{2} \\
& +\sum_{\text {dihedrals }} \frac{V_{n}}{2}[1+\cos (n \phi)] \\
& +\sum_{\text {nonbonded }}\left\{4 \varepsilon_{i j}\left[\left(\frac{\sigma_{i j}}{r_{i j}}\right)^{12}-\left(\frac{\sigma_{i j}}{r_{i j}}\right)^{6}\right]+\left[\frac{q_{i} q_{j}}{\varepsilon R_{i j}}\right]\right\}
\end{aligned}
$$




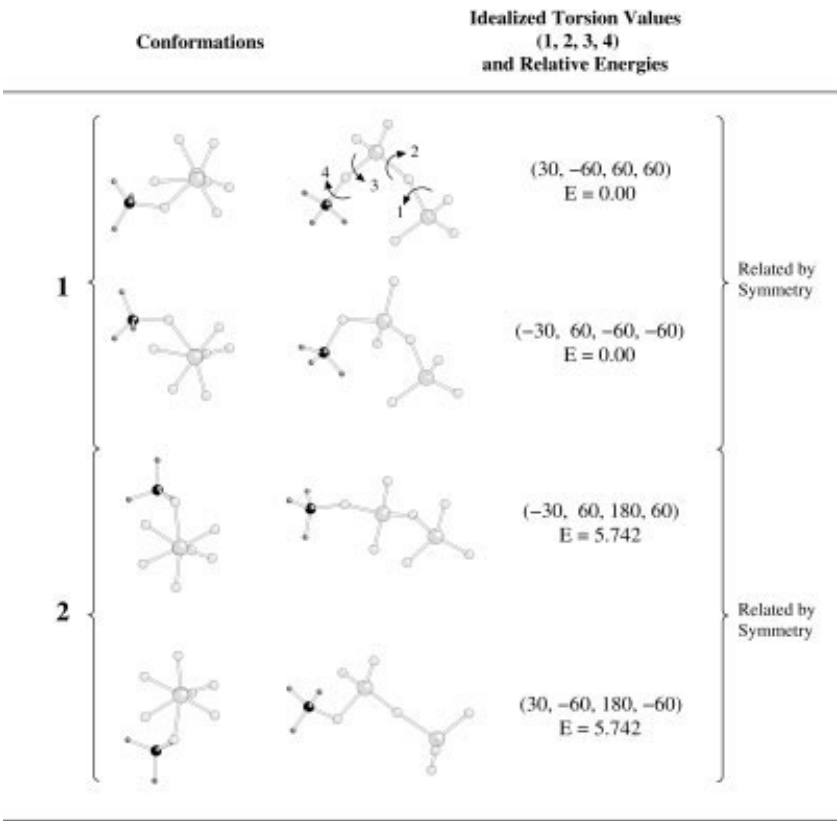

Figure 2. The local minima for MDP calculated at the RHF/6-31+G* level are provided. Relative energies are reported in $\mathrm{kcal} / \mathrm{mol}$, and conformers related through symmetry are noted.

where $\varepsilon_{i j}=\left(\varepsilon_{i} \varepsilon_{j}\right)^{1 / 2}, \sigma_{i j}=\left(\sigma_{i}+\sigma_{j}\right) / 2$, and 1-4 nonbonded terms are scaled by (1/1.2).

A primary goal was to introduce as few new parameters as possible, but the following parameters were necessary to fit the potential. A new atom type, $\mathrm{O} 3$, was needed to distinguish the unique conformational behavior of a terminal phosphate (see Fig. 1). The angle, bond, and Lennard-Jones parameters of $\mathrm{O} 3$ are the same as the $\mathrm{O} 2$ atom; only the partial charges and torsional parameters are different. No new bond parameters were necessary and most of the angle parameters were sufficient. It was not possible to properly fit the torsional parameters with the existing P-OS-P angle parameters, so a new force constant and equilibrium value were introduced. In addition to the $\mathrm{P}-\mathrm{OS}-\mathrm{P}$ angle, partial charges and torsional energy components $\left(V_{n}\right)$ were the focus of the parameterization. To verify our methodology described below, we properly reproduced the torsional parameters for a previously studied molecule, methyl phosphate, before parameterizing MDP and MTP (data not shown).

As outlined above, the potential energy surfaces of MDP and MTP were explored using ab initio molecular orbital calculations. For each of those conformations of the molecules (not just local minima), the Sander program ${ }^{11}$ was used to calculate the energetic contributions of the existing parameters within the AMBER force field (unknown force constants of the torsions and the P-OS-P angle were set to zero). The molecular mechanics contributions were calculated for all conformations from the scans of the potential surface. These relative energies were subtracted from the potential energy surface calculated with quantum mechanics methods. The resulting "torsional component" (including the P-OS-P angle contribution) was used to fit the missing parameters, $k_{\theta}$ and $V_{n}$. The equilibrium for the P-OS-P angle, $\theta_{o}$, was chosen as the average value for the $\mathrm{P}-\mathrm{OS}-\mathrm{P}$ angles from all the conformers of all scans.

The force constant for the P-OS-P angle and the torsional parameters were fit to the "torsional component" energy using an in-house, RMS-fitting program. The validity and independence of each parameter was examined through calculating the cross correlation between the fit variables. Crosscorrelation was minimal.

Initially, each torsional scan was fit individually to its particular slice of the potential surface, i.e., the $V_{n}$ for HC-CT-OS-P were fit only to the series of partial optimizations from scanning the $\mathrm{HC}-\mathrm{CT}-\mathrm{OS}-\mathrm{P}$ torsion in $10^{\circ}$ increments. This gave exceptional agreement between the molecular mechanics energies and those particular points on the RHF/6-31+ $\mathrm{G}^{*}$ potential surface (RMSD of $0.02-0.90 \mathrm{kcal} / \mathrm{mol}$ ), but these parameters yielded less accurate relative energies and conformations of the minima. Rather than fitting each torsion to a particular subset of points, the entire set—combining both MDP and MTP data—was fit simultaneously to reproduce the entire potential surface of both molecules. Some accuracy of individual potentials was lost to improve the overall performance of the parameters to reproduce the global behavior of the test molecules.

For each torsion, the inclusion of $V_{1}, V_{2}$, and $V_{3}$ energetic components was based on the symmetry of the "torsional energy" component in question. The minimal torsional components required to give accurate behavior are listed in Table 1. The exclusion of each $V_{n}$ component was verified through refitting the data with their inclusion and finding negligible improvement in the fit. The unnecessary components were also found to have parameter

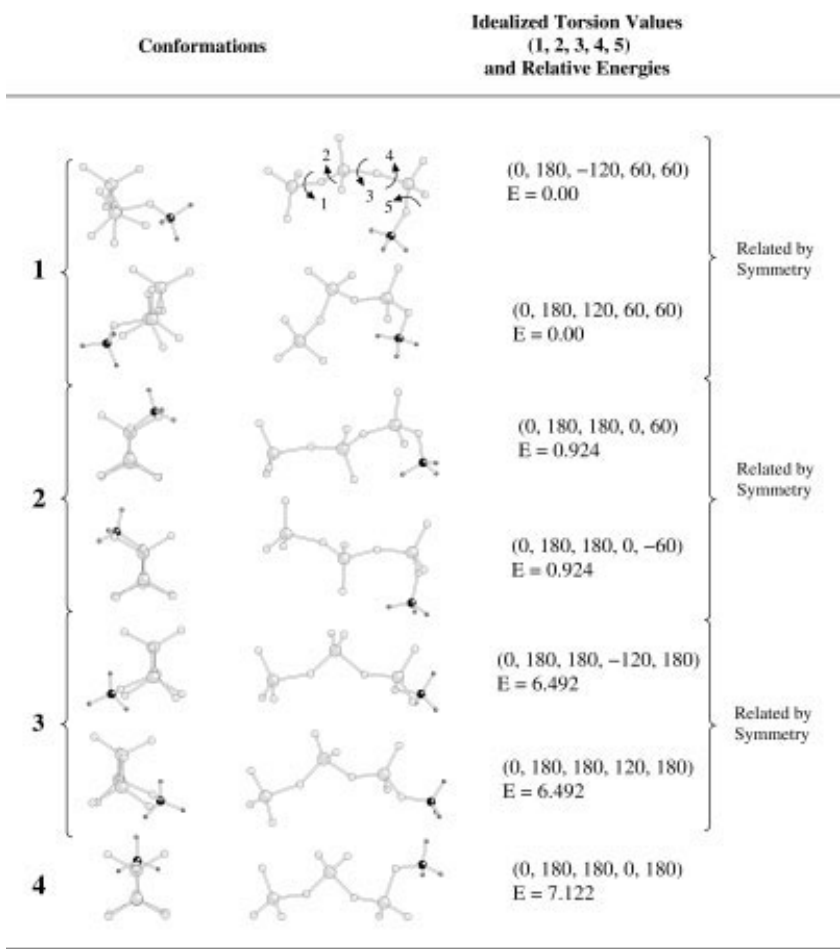

Figure 3. The local minima for MTP calculated at the RHF/6-31+G* level are provided. Relative energies are reported in $\mathrm{kcal} / \mathrm{mol}$, and conformers related through symmetry are noted. 
Table 1. The New Parameters to Supplement AMBER 94/99 Are Listed Below.

\begin{tabular}{lcc}
\hline Angle & $K_{\theta}{ }^{\mathrm{a}}$ & $\theta_{o}{ }^{\mathrm{b}}$ \\
\hline $\mathrm{P}-\mathrm{OS}-\mathrm{P}$ & 12.685 & 150.0
\end{tabular}

${ }^{\mathrm{a}} \mathrm{kcal} / \mathrm{mol} / \mathrm{radian}^{2}$

${ }^{\mathrm{b}}$ Degrees.

\begin{tabular}{lcrrr}
\hline Torsion & No. of paths & $V_{n} / 2^{\mathrm{d}}$ & $\gamma^{\mathrm{e}}$ & $n^{\mathrm{f}}$ \\
\hline HC-CT-OS-P & 3 & 0.105 & 0.00 & 3.0 \\
CT-OS-P-OS & 1 & -1.560 & 0.00 & 1.0 \\
CT-OS-P-O2 & 2 & 1.179 & 0.00 & -3.0 \\
CT-OS-P-O2 & 2 & -0.812 & 0.00 & 2.0 \\
P-OS-P-OS & 1 & 0.897 & 0.00 & 1.0 \\
P-OS-P-O2 & 2 & -0.709 & 0.00 & 2.0 \\
P-OS-P-O3 & 3 & -0.255 & 0.00 & 3.0
\end{tabular}

The parameters are repeated in the Supplemental Information using a different format appropriate for other force fields.

cNumber of bond paths that the total $V_{n} / 2$ is divided into.

${ }^{\mathrm{d}}$ Magnitude of torsion in $\mathrm{kcal} / \mathrm{mol}$.

${ }^{\text {e}}$ Phase offset in degrees.

${ }^{\mathrm{f}}$ The periodicity of the torsion.

values close to zero, physically unrealistic parameter values, high uncertainty, and/or large crosscorrelation to the other parameters.

\section{Verifying the Accuracy and Transferability of the Parameters}

In addition to fitting the entire potential surface, the parameter sets were further tested for the proper reproduction of conformations and relative energies of both the MDP and MTP local minima. The conformations of the molecules in each local minimum on the $\mathrm{RHF} / 6-31+\mathrm{G}^{*}$ potential surface were used as the starting points for energy minimization in Sander. Minimizations were conducted using the new parameters, and minimizations were repeated with the existing, estimated parameters in AMBER 94/99. Estimated torsional parameters exist in the AMBER 94/99 force field; however, partial charges for di- and triphosphates do not. To evaluate the new torsional parameters, the RESP charges calculated in this work were used in conjunction with the estimated torsional parameters to give values referred to as "existing AMBER parameters" (so "existing" parameters are a necessary hybrid of new partial charges and existing torsional and angle potentials). These minima and their relative energies were compared to the quantum mechanics calculations.

Transferability of the parameters was evaluated by determining minima for inorganic diphosphate and triphosphate. The minima based on our new parameters for AMBER were compared to minima determined with $\mathrm{RHF} / 6-31+\mathrm{G}^{*}$ calculations. Minima were also determined using the existing estimated parameters in the AMBER 94/99 force field. Again, the partial charges in the existing parameter case came from our $a b$ initio calculations.

To examine the transferability of these parameters to condensed-phase systems, four proteins complexed with ATP were chosen (1F2U, 1F9A, 1GOL, 1NSF). These structures contained only protein, ATP, magnesium ions, and water molecules. This avoids any unusual ligands, salts, or cofactors that may not be covered by the current force fields. Hydrogens were added to the system using Leap and minimized using Sander. The ATP residue (and all hydrogen atoms in the system) was then minimized with the Sander program until convergence or a maximum of 50,000 cycles was reached. The final minimized ATP conformations were compared to the initial crystal structures. This procedure was repeated using the existing, estimated parameters in the AMBER 94/99 force field as well.

To provide a comparison of the new parameters to other standard molecular mechanics force fields, the same minimizations of four protein-ligand complexes were completed using the CHARMM force field ${ }^{4}$ and the CHARMM program. ${ }^{12}$

\section{Results and Discussion}

\section{New Parameters}

Partial charges and atom types for MDP and MTP are given in Figure 1. The new polyphosphate parameters for use with the AMBER 94/99 force field are given in Table 1. The new parameters have been fit with excellent precision to the potential surfaces for MDP and MTP. The overall RMSD to the potential surface for the new parameters is $0.62 \mathrm{kcal} / \mathrm{mol}$. The agreement is well below the typical goal of ca. $1.0 \mathrm{kcal} / \mathrm{mol}$, and the agreement is even more impressive when compared to the existing, estimated AMBER parameters which give an RMSD of $7.8 \mathrm{kcal} / \mathrm{mol}$.

\section{Evaluation of the Conformations and Relative Energies}

The local minima of MDP and MTP as calculated at the RHF/ $6-31+\mathrm{G}^{*}$ level are given in Figures 2 and 3. Many of the conformers were related through symmetry; there were two unique conformers for MDP and four for MTP. Coordinates for the various conformers are given in the Supplemental Information.

The parameters must be evaluated for the ability to produce proper conformational behavior and relative energies. Figures 4 and 5 compare the local minima for MDP and MTP from minimizations using the new polyphosphate parameters vs. the existing estimates in the AMBER force field. In comparison to the minima from the quantum mechanics calculations, the conformations of MDP are greatly improved with the new parameters. The average RMSD using the new parameters is $0.53 \AA$ compared to $1.73 \AA$ for the existing AMBER parameters. This RMSD is calculated solely for the phosphate portion of the molecule. Due to the modular nature of the AMBER force field, the methyl group will be replaced when these parameters are used in polyphosphorylated nucleotides. Therefore, it is illustrative to focus on the RMS of the phosphate moiety. The new parameters also rank the two conformers in the correct order, closely predicting the $5.7 \mathrm{kcal} / \mathrm{mol}$ barrier due to the favorable intramolecular "hydrogen bond." The existing AMBER potential ranks both conformers at the same energy, failing to distinguish the importance of the intramolecular interaction.

The new parameter set also provides appropriate conformations and relative energies for MTP. The new parameters produce conformers with an average RMSD of $0.60 \AA$ to the quantum calcu- 


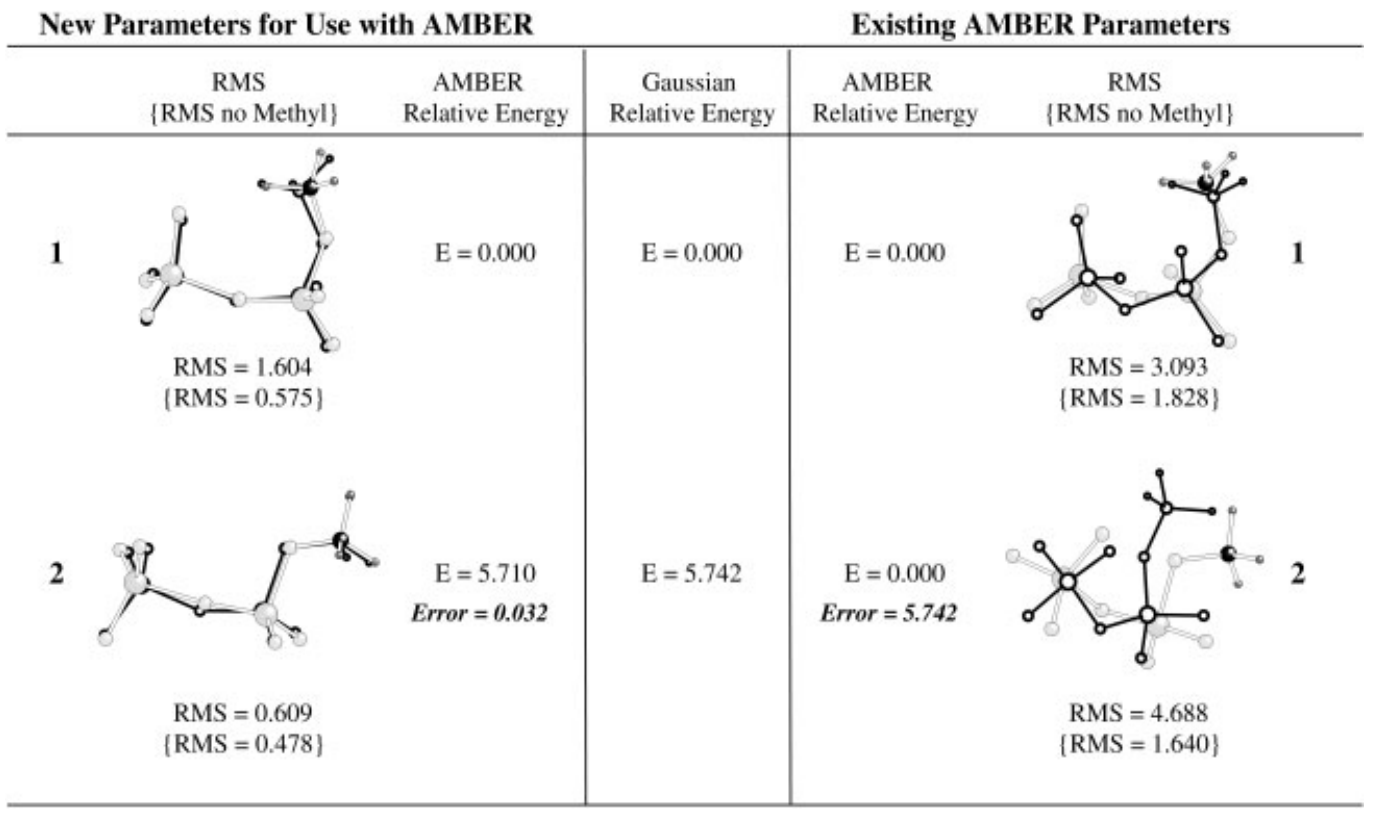

Figure 4. The ability of the AMBER parameters to reproduce of the local minima of MDP is evaluated. Conformers determined with RHF/6-31+ $\mathrm{G}^{*}$ calculations are shown in grayscale (ball and stick). The conformers calculated with AMBER are shown in black (ring and stick). The local minima are superimposed to highlight agreement. The RMS deviation between the atoms is given for the whole molecule and for the phosphate portion without the methyl group. Energies are given in $\mathrm{kcal} / \mathrm{mol}$.

lations while the existing parameters have an average RMSD of $2.77 \AA$ (focusing on the atoms of the phosphate group). The new parameters enable the four unique MTP conformers to be energetically ranked in the correct order. The intramolecular "hydrogen bond" in MTP is still significant $(5.22 \mathrm{kcal} / \mathrm{mol})$, but underestimated as the barrier is $7.12 \mathrm{kcal} / \mathrm{mol}$ in the $\mathrm{RHF} / 6-31+\mathrm{G}^{*}$ calculations. However, it is a marked improvement over the existing AMBER parameters, which fail to identify the large energy difference by more than $4 \mathrm{kcal} / \mathrm{mol}$. The existing AMBER parameters also fail to correctly order the relative energies of the conformers.

\section{Evaluation of the Potential Surface}

The ability of the new parameters to reproduce of the torsional scans from the RHF/6-31+G* calculations is shown in Figures 6 and 7 . Scanning the surface with the existing parameters gave very poor results (data not shown, but recall that the potential surface had an RMSD of $7.8 \mathrm{kcal} / \mathrm{mol}$ to the RHF/6-31+ $\mathrm{G}^{*}$ surface). The potential surface of the new parameters is able to reproduce the fine detail of the MDP potential surface (Fig. 6A and B) as well as the large global conformational changes. In Figure $6 \mathrm{C}$, the incongruous nature of the curve is due to the gross conformational and energetic changes that accompany the formation and dissolution of the intramolecular "hydrogen bond." There is a discrepancy in the minima for the H-CT-OS-P torsion, which can be seen in Figure $6 \mathrm{~A}$. Introducing an offset of $90^{\circ}$ into the torsional term improved the fit for that subset of points; however, the overall accuracy of the fit of the entire surface decreased and the resulting parameters extended poorly to MTP, which did not require an offset. The small discrepancy in Figure 6A was acceptable given the improved transferability of the parameter.

The reproduction of the MTP potential is also quite remarkable. The new parameters fit the potential surface well, both reproducing the position of the minima and the height of the barriers. Similar to the MDP case, Figure 7F shows an incongruity due to the large conformational change induced by the intramolecular "hydrogen bond." In MTP there are three unique P-OS-P-OS torsions, all of which are represented with a single parameter. The new parameter set fits the fine topology of the P-OS-P-OS (2) exceptionally well (Fig. 7D). The fit is good for $\mathrm{P}-\mathrm{OS}-\mathrm{P}-\mathrm{OS}$ (1) and $\mathrm{P}-\mathrm{OS}-\mathrm{P}-\mathrm{OS}$ (3), but the maxima are off by approximately $1 \mathrm{kcal} / \mathrm{mol}$ (Fig. 7C and $\mathrm{E}$, respectively). These discrepancies are necessary to improve the global performance of the parameters. Overall, the new parameters for use with the AMBER force field accurately reproduce the location of local minima, the height of rotational barriers, and the curvature of the potential surfaces of MDP and MTP.

\section{Parameterization of the P-OS-P Angle}

Visualization of the normal modes of the minima of MDP and MTP (calculated with Gaussian 98), revealed a particularly soft normal mode $\left(49.3 \mathrm{~cm}^{-1} \text { and } 85.6 \mathrm{~cm}^{-1}\right)^{13}$ for the P-OS-P angles in both polyphosphates. This has been noted previously by Hwang et al. ${ }^{8}$ and in the parameterization of polyphosphates for the CHARMM force field. ${ }^{4}$ The estimated P-OS-P angle parameters in the AMBER force field appear to have been taken by analogy from the CT-OS-P angle; however, the normal modes that involve the CT-OS-P bend have much higher frequencies in these molecules $\left(198.7\right.$ and $282.1 \mathrm{~cm}^{-1}$ in 


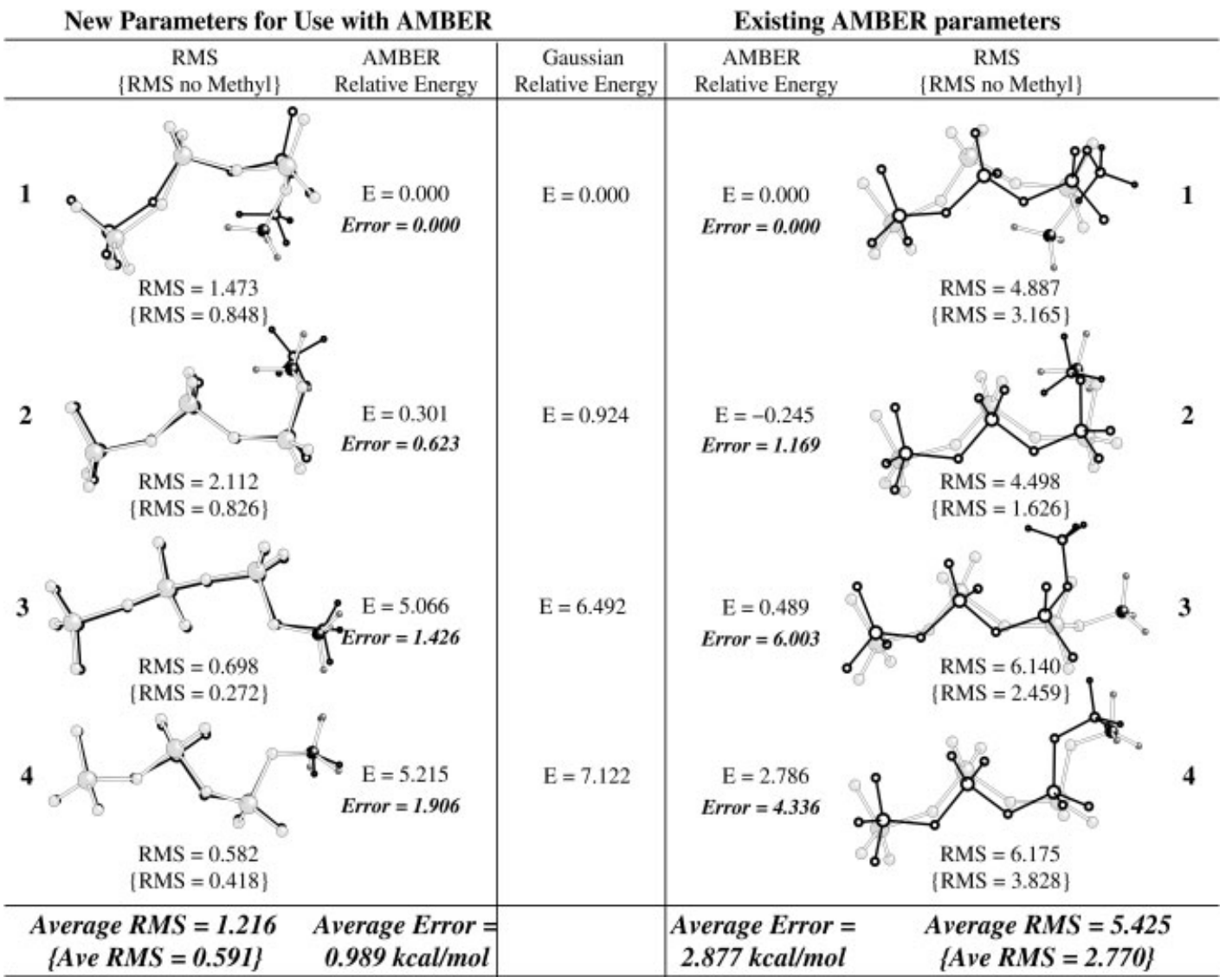

Figure 5. The ability of the AMBER parameters to reproduce of the local minima of MTP is evaluated. Conformers determined with $\mathrm{RHF} / 6-31+\mathrm{G}^{*}$ calculations are shown in grayscale (ball and stick). The conformers calculated with AMBER are shown in black (ring and stick). The local minima are superimposed to highlight agreement. The RMS deviation between the atoms is given for the whole molecule and for the phosphate portion without the methyl group. Energies are given in $\mathrm{kcal} / \mathrm{mol}$.

the frequency calculations using Gaussian 98). ${ }^{13}$ The differences in the angle bending make this comparison suspect. The charged phosphate groups have more diffuse electron clouds, giving the P-OS-P angle less "organic" character than the CT-OS-P. Such an angle should have different properties than its carbon analog.

The existing P-OS-P and CT-OS-P angle parameters consist of an equilibrium angle of $120.5^{\circ}$ and a force constant of 100 $\mathrm{kcal} / \mathrm{mol} / \mathrm{rad}$. The equilibrium angle is too small for accurate parameterization. Previous DFT calculations of di- and triphosphates chelated with magnesium and calcium ions indicate that the lowest energy conformers have near-linear P-OS-P angles. ${ }^{14}$ Studies of the P-OS-P angle based on surveys of crystal structures have found that the equilibrium angle is approximately $130^{\circ} .4,15,16$ Our torsional scans of the RHF/6-31+G* surface revealed that the $\mathrm{P}-\mathrm{OS}-\mathrm{P}$ equilibrium angle averaged $150^{\circ}$. In fitting our parameters to that potential surface, the equilibrium value was set to the average of $150^{\circ}$. This is in good agreement with the CHARMM force field. ${ }^{4}$ The CHARMM parameters have a P-OS-P equilibrium angle of $140^{\circ}$ and a Urey-Bradley 1,3 stretching term for the $\mathrm{P}-\mathrm{OS}-\mathrm{P}$ angle. Figure 8B shows the P-OS-P angle term as a sum of the angle bending and Urey-Bradley terms from the CHARMM parameters. The combination of the two terms leads to an apparent equilibrium angle of $160^{\circ}$.

The high force constant $(100 \mathrm{kcal} / \mathrm{mol} / \mathrm{rad})$ of the existing parameters is not consistent with the low frequency of the normal modes involving of the P-OS-P angle. Over the course of our torsional scans, the $\mathrm{P}-\mathrm{OS}-\mathrm{P}$ angle ranged from $140^{\circ}$ to $160^{\circ}$. With the existing parameters, this $20^{\circ}$ change in the P-OS-P angle-far from the equilibrium value of $120.5^{\circ}$-resulted in a $40-\mathrm{kcal} / \mathrm{mol}$ change in energy (see Fig. 8A)! This unrealistic energetic penalty is the dominating factor in the failure of the existing AMBER force field to accurately model the behavior of polyphosphates. The new parameter set has a P-OS-P force constant of $12.685 \mathrm{kcal} / \mathrm{mol} /$ $\mathrm{rad}^{2}$. This leads to much smaller energy penalties as the P-OS-P angle varies (Fig. 8A), and is in good agreement with the CHARMM P-OS-P angle-bending force constant of $15 \mathrm{kcal} / \mathrm{mol} / \mathrm{rad}^{2}$ (Fig. 8B).

\section{Reproduction of Conformations of Inorganic Polyphosphates}

Good parameters should be generally transferable and able to reproduce conformational behavior of additional polyphosphates, 
not just the molecules explicitly studied. Therefore, the parameters were used to reproduce the minima of inorganic di- and triphosphate (DP and TP, respectively). Figure 9 shows the improved ability of the new parameters to produce conformations of TP and DP in good agreement with RHF/6-31+G* calculations. Also shown is a comparison of the ability of the new parameters and existing parameters to fit the potential surface of TP and DP at the RHF/6-31+G* level (Fig. 10). The existing AMBER parameters are unable to accurately model the behavior of the inorganic polyphosphates, erroneously creating large barriers to the rotation about the O3-P-OS-P torsion (over $4 \mathrm{kcal} / \mathrm{mol}$ in Fig. 10D) and the P-OS-P-OS torsion (20 kcal/mol in Fig. 10B)! Similarly, the $\mathrm{P}-\mathrm{OS}-\mathrm{P}-\mathrm{O} 3$ torsion in DP is exaggerated by over $15 \mathrm{kcal} / \mathrm{mol}$ (Fig. 10F). Also, the stiff nature of the existing P-OS-P angle causes an apparent loss of threefold symmetry in the O3-P-OS-P torsion when using the existing AMBER parameters. The new parameters provide a significant improvement for modeling the behavior of inorganic polyphosphates.

\section{Reproduction of Conformations of Protein-Bound ATP}

The new parameters were also used to minimize phosphorylated nucleotides in a condensed-phase, protein environment. Four complexes containing ATP and a coordinating $\mathrm{Mg}^{+2}$ ion were chosen from the PDB (1F2U, 1F9A, 1GOL, 1NSF). Hydrogens were added to the structures and initial minimizations allowed the hydrogens to relax to resolve any steric clashes. Next, the ATP residue and the hydrogen atoms were allowed to minimize within the protein environment. The minimizations were carried out using both the new parameters as well as the existing parameters.
A

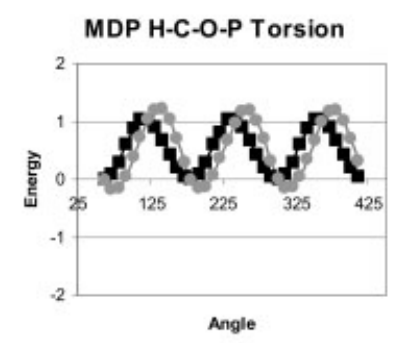

C
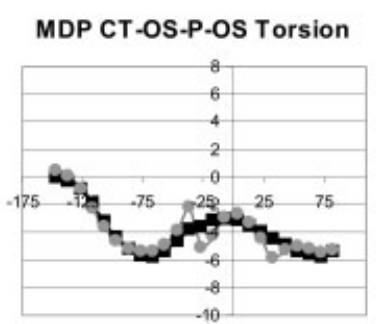

B
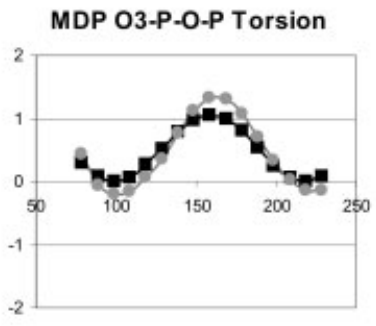

D

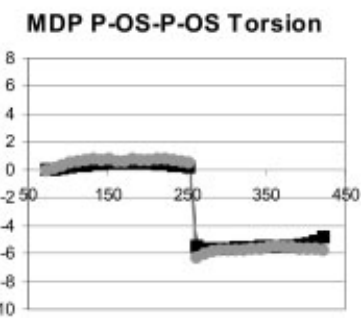

Figure 6. Reproduction of the RHF/6-31+G* potential surface for MDP. Relative energies (kcal/mol) for RHF/6-31+ $\mathrm{G}^{*}$ (black squares) and AMBER 94/99 with the new parameters (gray circles) are plotted vs. torsional angle. Two scales are used to show both the fine details $(-2$ to $+2 \mathrm{kcal} / \mathrm{mol})$ of parameter agreement and the ability to reproduce the gross conformational change associated with the intramolecular hydrogen bond interaction $(-10$ to $+8 \mathrm{kcal} / \mathrm{mol})$.
A

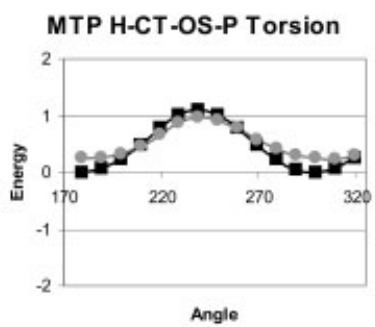

C
MTP P-OS-P-OS (1)

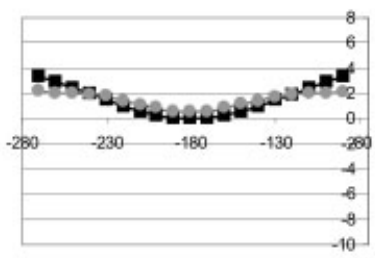

E

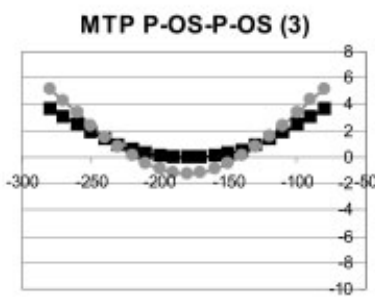

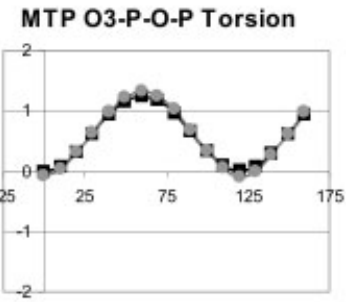

D

MTP P-OS-P-OS (2)
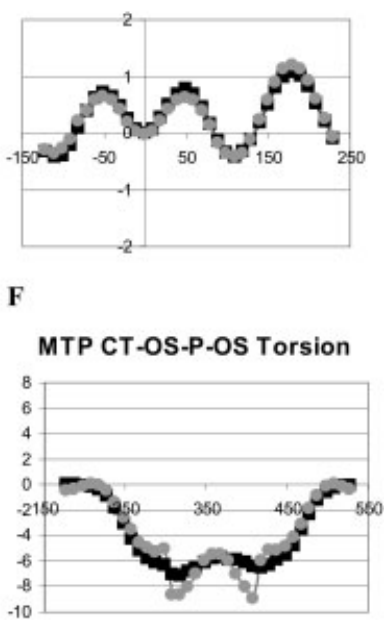

Figure 7. Reproduction of the RHF/6-31+ $\mathrm{G}^{*}$ potential surface for MTP. Relative energies $(\mathrm{kcal} / \mathrm{mol})$ for $\mathrm{RHF} / 6-31+\mathrm{G}^{*}$ (black squares) and AMBER 94/99 with the new parameters (gray circles) are plotted vs. torsional angle. Two scales are used to show both the fine details of parameter agreement and the ability to reproduce the gross conformational change associated with the intramolecular hydrogen bond interaction.

Table 2 presents the RMSD values for the minimized structures compared to the original position of ATP in the crystal structure. Surprisingly, there is little difference seen in the minimized positions for ATP using the new parameters (the average RMSD for the new parameters is slightly lower, but negligibly so). The most probable reason is that the agreement in most cases is already very good, well below $1 \AA$. It is difficult to improve on near-perfect agreement. Another possible reason that the results with the new parameters are so similar to the existing parameters is because the same partial charges were used for both the existing and new parameter sets. The electrostatic interactions between the highly charged ATP and the magnesium ions dominate the behavior, and so using the same partial charges in both parameter sets creates nearly identical conformations of ATP. The only difference between the two parameter sets is the torsional and angle parameters, but those contributions are small in comparison to coordination to a divalent ion.

However, improvement is seen in some of the structural details of the two minimizations. The P-OS-P angles obtained with the minimizations using the new parameters are in better agreement with the P-OS-P angles measured in the crystal structure. Table 3 
A

\section{Comparison of AMBER P-O-P Angle Parameters}

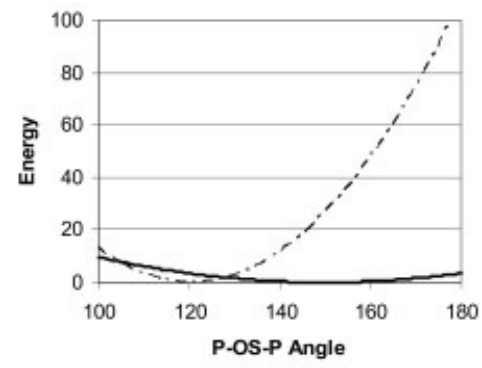

B

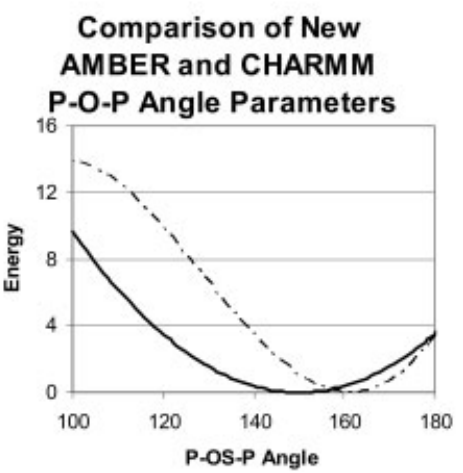

Figure 8. (A) Comparison of existing and new $\mathrm{P}-\mathrm{OS}-\mathrm{P}$ angle parameters for use with AMBER. The existing P-OS-P parameters $\left(k_{\theta}=\right.$ $100, \theta_{o}=120.5^{\circ}$ ) show a dramatic $40-\mathrm{kcal} / \mathrm{mol}$ energy change between $140^{\circ}$ and $160^{\circ}$ (dashed line). In comparison, the new P-OS-P parameters show little change (solid line). (B) Comparison of the new $\mathrm{P}-\mathrm{OS}-\mathrm{P}$ angle parameters for use with AMBER and the parameters for the same angle from CHARMM. ${ }^{4}$ Good agreement is seen for the equilibrium angle and force constant despite slightly different functional forms for the energy in the two force fields. Note that the scale for energies is not the same in (A) and (B).

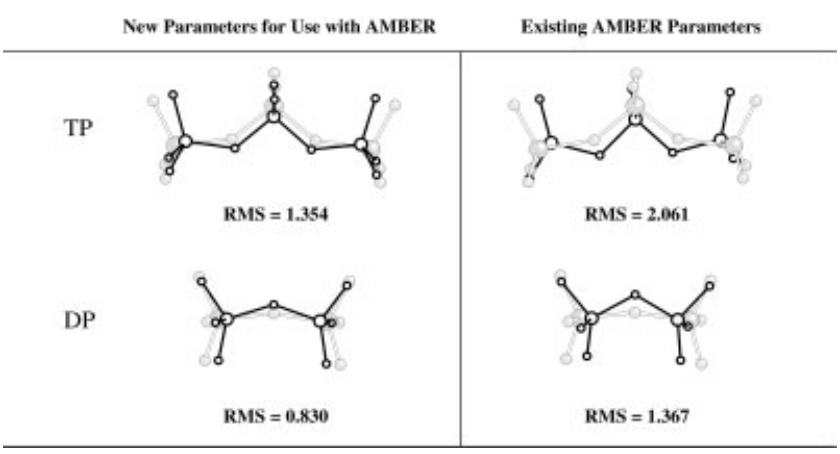

Figure 9. Extrapolating the new parameters to inorganic test systems. The conformers determined with $\mathrm{RHF} / 6-31+\mathrm{G}^{*}$ calculations (grayscale, ball and stick) are compared to the conformers determined with AMBER using the existing and new parameters (black, ring and stick). The new parameters have better agreement with the minima determined with quantum mechanics calculations.
A

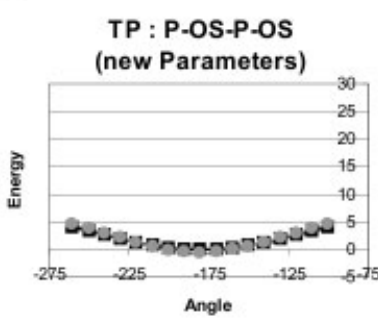

C

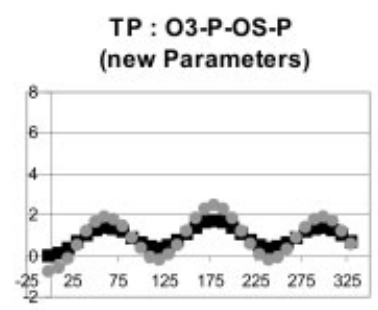

E

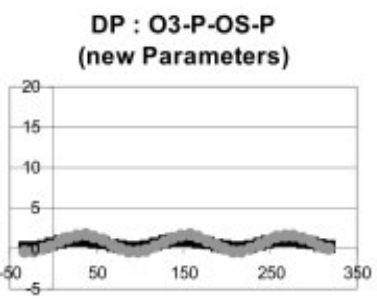

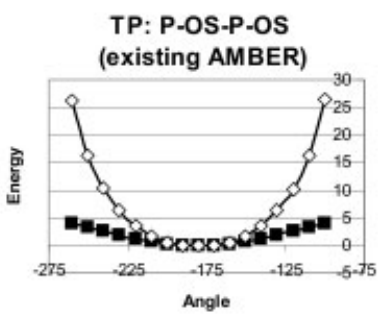

D

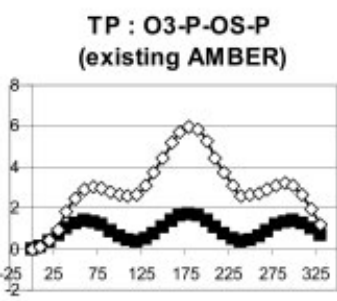

$\mathbf{F}$

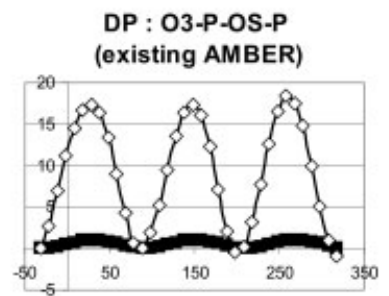

Figure 10. Reproduction of the RHF/6-31+ $\mathrm{G}^{*}$ potential surface for TP and DP. Relative energies $(\mathrm{kcal} / \mathrm{mol}$ ) for RHF/6-31+G* (black squares) are plotted vs. torsional angle. The energies of the same conformations using the new parameters are given in (A), (C), and (E) (gray circles), and the results for the existing AMBER parameters are given in (B), (D), and (F) (open diamonds).

shows that the new parameters reproduce the crystal structure $\mathrm{P}-\mathrm{OS}-\mathrm{P}$ angles within $2^{\circ}$, whereas the existing parameters consistently underestimate the $\mathrm{P}-\mathrm{OS}-\mathrm{P}$ angle by an average of $12^{\circ}$. The new parameters could improve modeling of molecular recognition because it directly influences the position of the highly charged OS atom between the phosphate groups.

Although the minima are very similar for the two datasets, the energies observed during the minimizations are different (Table 4).

Table 2. The Agreement between the Positions of ATP in the Crystal Structures Compared to the Minima Using Both Sets of AMBER Parameters (units are in $\AA$ ).

\begin{tabular}{lcc}
\hline & \multicolumn{2}{c}{ RMSD } \\
\cline { 2 - 3 } PDB Code & New Parameters & Existing Parameters \\
\hline 1F2U & 0.23 & 0.25 \\
1F9A & 0.55 & 0.52 \\
1GOL & 1.67 & 1.67 \\
1NSF & 0.29 & 0.33 \\
\end{tabular}


Table 3. The Two P-OS-P Angles in ATP Were Measured for the Crystal Structure and for the Minima Determined with Both AMBER Parameter Sets.

\begin{tabular}{lccccc}
\hline & & New & Old & Error New & $\begin{array}{c}\text { Error Old } \\
\text { Parameters }\end{array}$ \\
\hline \multirow{2}{*}{ 1F2U } & 133.2 & 129.0 & 121.7 & -4.2 & -11.5 \\
& 133.5 & 135.2 & 123.6 & 1.7 & -9.9 \\
1F9A & 135.4 & 126.8 & 120.2 & -8.6 & -15.2 \\
& 135.3 & 146.4 & 122.4 & 11.1 & -12.9 \\
\multirow{2}{*}{ 1GOL } & 131.3 & 123.3 & 119.5 & -8 & -11.8 \\
& 128.9 & 124.3 & 119.1 & -4.6 & -9.8 \\
\multirow{2}{*}{ 1NSF } & 134.7 & 129.1 & 120.8 & -5.6 & -13.9 \\
& 131.4 & 131.2 & 120.6 & -0.2 & -10.8 \\
\multirow{2}{*}{ Average } & $\mathbf{1 3 3 . 0}$ & $\mathbf{1 3 0 . 7}$ & $\mathbf{1 2 1 . 0}$ & $\mathbf{- 2 . 3}$ & $\mathbf{- 1 2 . 0}$ \\
& & & & & \\
\hline
\end{tabular}

The new parameters are in better agreement with the crystal structure.

Most notably, the new parameter set produces lower relative energies for the original conformation of ATP in the crystal structure. The new parameters result in an energy difference between the starting and final conformations that is approximately 10 $\mathrm{kcal} / \mathrm{mol}$ smaller than the energies using the existing parameters. This means that conformations resembling the crystal structure are more likely to be sampled in an MD simulation using the new parameters. Normally, one would consider energy differences of $100 \mathrm{kcal} / \mathrm{mol}$ or more to be too large to be sampled in a simulation, but the reader must remember that the small, thermal motions of the highly charged ATP and $\mathrm{Mg}^{+2}$ in an MD simulation will produce these large energy changes. We expect that the parameters
Table 4. Energies Observed during the AMBER Minimizations of the Protein-ATP Complexes.

\begin{tabular}{llccr}
\hline PDB Code & Parameters & Initial Energy & Final Energy & $\Delta E$ \\
\hline \multirow{2}{*}{ 1F2U } & New & -64215 & -64298 & -83 \\
& Existing & -64203 & -64295 & -92 \\
1F9A & New & -20419 & -20680 & -261 \\
& Existing & -20400 & -20678 & -278 \\
1GOL & New & -36689 & -37437 & -748 \\
& Existing & -36678 & -37435 & -757 \\
1 NSF & New & -27303 & -27368 & -65 \\
& Existing & -27291 & -27368 & -77 \\
\hline
\end{tabular}

Only ATP and the hydrogens were allowed conformational freedom during the minimizations. The new parameters consistently produce smaller energy differences between the starting conformation from the crystal structure and the final conformation.

will result in improved sampling of ATP and similar polyphosphorylated molecules in protein simulations.

\section{Validation of the New Parameters through Comparison to CHARMM}

It was important to compare the performance of the new parameters to other well-cited force fields from the literature. Because the new P-OS-P angle compares nicely to the CHARMM force field, it was most appropriate to compare the performance of our improved AMBER force field to CHARMM.

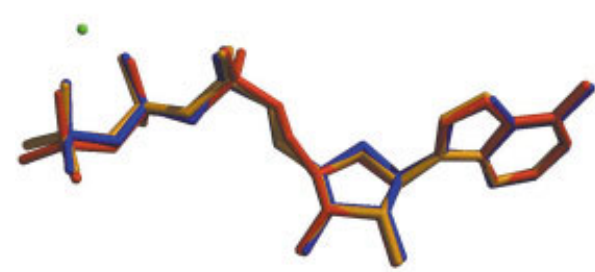

$1 F 2 U$

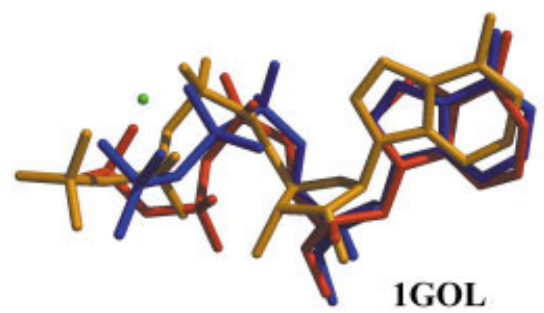

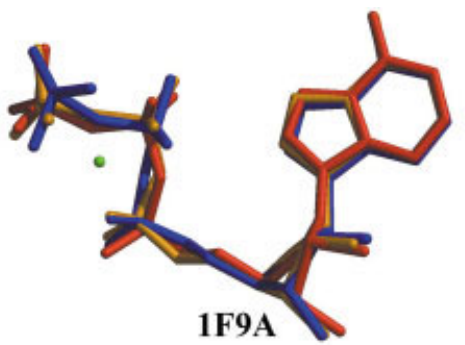

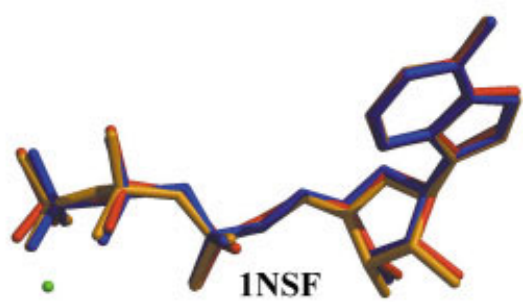

Figure 11. The minimized ATP molecules are compared to their crystallographic positions. The overlay is based on a RMS fit of the protein backbone before and after minimization of the hydrogens and the ATP. The crystal conformation is shown in red, and the minima using the new AMBER parameters are shown in gold. The minima resulting from the CHARMM parameters are shown in blue. The adjacent magnesium ion is shown in green. The agreement between the new parameters, CHARMM, and the crystal structures verifies that the parameterization presented in this work is robust. 
The new parameters will most likely be used in the modeling of polyphosphorylated molecules bound to proteins, and we wished to compare the force fields in this setting. The four systems of protein-bound ATP were minimized using the CHARMM force field $^{4}$ and the CHARMM program. ${ }^{12}$ As can be seen in Figure 11, the positions and conformations of ATP are almost identical for our new parameters and CHARMM. This verifies that our results are in keeping with other established force fields widely used in the field.

\section{Conclusions}

Our new parameters provide exceptional agreement with the minima and torsional scans of the RHF/6-31+ G* potential surface for MDP, MTP, DP, and TP. The new parameters are a significant improvement over the existing estimates in AMBER 94/99, and extend well to test systems. We were surprised to find that the minima of protein-bound ATP were nearly the same with the new and existing parameters. However, most of the minima were in excellent agreement with the crystal structure, and it is difficult to improve an already good result. With the new parameters, the $\mathrm{P}-\mathrm{OS}-\mathrm{P}$ angles were in better agreement with the crystal structure, and the energies indicate that the new parameters could lead to more extensive sampling of the potential surface during simulations of polyphosphorylated molecules. Lastly, the new parameters for use with AMBER 94/99 provide results for protein-bound ATP that are in excellent agreement with CHARMM, a well-respected force field used extensively in the computational community.

\section{Supplementary Material}

The parameters are given in an alternate format for use with other force fields like OPLS. A frcmod file is provided for AMBER users. The coordinates for the RHF/6-31+G* minima are also provided.

\section{Acknowledgments}

We would like to thank Prof. Charles L. Brooks, III, for his assistance with the CHARMM calculations. We greatly appreciate his help with the protein-bound ATP systems. We would like to thank Prof. William L. Jorgensen and Dr. D. C. Lim for their generous donation of the ChemEdit program used to generate many of the figures. H.A.C. is grateful for an award through the Beckman Young Investigator Program. K.L.M. is grateful for receiving fellowships from Edward S. Blake, Fred. W. Lyons, American Federation for Pharmaceutical Education, and the University of Michigan Regents. The contents are solely the responsibility of the authors and do not necessarily represent the official views of the NIGMS.

\section{References}

1. Halgren, T. A.; Damm, W. Curr Opin Struct Biol 2001, 11, 236.

2. Cornell, W. D.; Cieplak, P.; Bayly, C. I.; Gould, I. R.; Merz, K. M.; Ferguson, D. M.; Spellmeyer, D. C.; Fox, T.; Caldwell, J. W.; Kollman, P. A. J Am Chem Soc 1995, 117, 5179.

3. Petrova, P.; Koca, J.; Imberty, A. J Am Chem Soc 1999, 121, 5535.

4. Pavelites, J. J.; Gao, J.; Bash, P. A.; Mackerell, A. D. J Comput Chem 1997, 18, 221.

5. Stewart, E., L.; Nevins, N.; Allinger, N. L.; Bowen, J. P. J Org Chem $1999,64,5350$

6. Vetter, I. R.; Wittinghofer, A. Q Rev Biophys 1999, 1, 1

7. Brady, A. E.; Limbird, L. E. Cellular Signalling, 2002, 14, 297.

8. Hwang, M.-J.; Chu, P.-Y.; Chen, J.-C.; Chao, I. J Comput Chem 1999, 20, 1702 .

9. Frisch, M. J.; Trucks, G. W.; Schlegel, H. B.; Scuseria, G. E.; Robb, M. A.; Cheeseman, J. R.; Zakrzewski, V. G.; Montgomery J. A., Jr.; Stratmann, R. E.; Burant, J. C.; Dapprich, S.; Millam, J. M.; Daniels, A. D.; Kudin, K. N.; Strain, M. C.; Farkas, O.; Tomasi, J.; Barone, V.; Cossi, M.; Cammi, R.; Mennucci, B.; Pomelli, C.; Adamo, C.; Clifford, S.; Ochterski, J.; Petersson, G. A.; Ayala, P. Y.; Cui, Q.; Morokuma, K.; Malick, D. K.; Rabuck, A. D.; Raghavachari, K.; Foresman, J. B.; Cioslowski, J.; Ortiz, J. V.; Baboul, A. G.; Stefanov, B. B.; Liu, G.; Liashenko, A.; Piskorz, P.; Komaromi, I.; Gomperts, R.; Martin, R. L.; Fox, D. J.; Keith, T.; Al-Laham, M. A.; Peng, C. Y.; Nanayakkara, A.; Challacombe, M.; Gill, P. M. W.; Johnson, B.; Chen, W.; Wong, M. W.; Andres, J. L.; Gonzalez, C.; Head-Gordon, M.; Replogle, E. S.; Pople, J. A. Gaussian 98, Revision A.9; Gaussian, Inc.: Pittsburgh, PA, 1998.

10. Cieplak, P.; Cornell, W. D.; Bayly, C.; Kollman, P. A. J Am Chem Soc 1995, 16, 1357.

11. Case, D. A.; Pearlman, D. A.; Caldwell, J. W.; Cheatham T. E., III; Ross, W. S.; Simmerling, C. L.; Darden, T. A.; Merz, K. M.; Stanton, R. V.; Cheng, A. L.; Vincent, J. J.; Crowley, M.; Tsui, V.; Radmer, R. J.; Duan, Y.; Pitera, J.; Massova, I.; Seibel, G. L.; Singh, U. C. Weiner, P. K.; Kollman, P. A. AMBER 6; University of California San Francisco: San Francisco, CA, 1996.

12. Brooks, B.; Bruccoleri, B.; Olafson, D.; States, D.; Swaminathan, S. Karplus, M. J Comput Chem 1983, 4,187.

13. These frequencies have been scaled by 0.89 .

14. Cini, R.; Chindamo, D.; Catenaccio, M.; Lorenzini, S.; Marcolongo, R. J Biomol Struct Dynam 2000, 18, 155.

15. Mandel, M. S. Acta Crystallogr B 1975, B31, 1730.

16. Tvaroska, I.; Andre, I.; Carver, J. P. J Mol Struct (Theochem) 1999, 469, 103 . 